\title{
Orthocentric Systems in Minkowski Planes
}

\author{
Tobías Rosas Soto (tjrosas@hotmail.com) \\ Wilson Pacheco Redondo (wpachecoredondo@gmail.com) \\ Departamento de Matemática \\ Facultad Experimental de Ciencias \\ Universidad del Zulia \\ Venezuela
}

April 2, 2019

\begin{abstract}
A new way to define the notion of $\mathcal{C}$-orthocenter will be displayed by studying some propierties of four points in the plane which allows to extend the notion of Euler's line, the Six Point Circles and the three-circles theorem, for normed planes. In the present paper (which can be regarded as extention of 10 in Minkowski plane in general) we derive several characterizations of the Euclidianity of the plane.

Key words: $\mathcal{C}$-Orthocenters, $\mathcal{C}$-Orthocentryc systems, Minkowski planes, Birkhoff orthogonality, Isosceles orthogonality, Euclidianity.
\end{abstract}

\section{Introduction}

By $(M,\|\circ\|)$ we denote an arbitrary Minkowski plane (i.e., a real two-dimensional normed linear space) with unit circle $\mathcal{C}$, origin $O$, and norm $\|$ o $\|$. Basic references to the geometry of Minkowski planes are [7], [9, [12] and the monograph [11]. For any point $x \in(M,\|\circ\|)$ and any number $\lambda>0$, the set $\mathcal{C}(x, \lambda):=x+\lambda \mathcal{C}$ is said to be the circle centered at $x$ and having radius $\lambda$. It has been shown by E. Asplund and B. Grünbaum in [3] that the following theorem, which is the extension of the classical three-circles theorem in the euclidean plane, also holds in strictly convex planes.

Theorem. If three circles $\mathcal{C}\left(x_{1}, \lambda\right), \mathcal{C}\left(x_{2}, \lambda\right)$, and $\mathcal{C}\left(x_{3}, \lambda\right)$ pass through a common point $p_{4}$ and intersect pairwise in the points $p_{1}, p_{2}$, and $p_{3}$, then there exists a circle $\mathcal{C}\left(x_{4}, \lambda\right)$ such that $\left\{p_{1}, p_{2}, p_{3}\right\} \subseteq \mathcal{C}\left(x_{4}, \lambda\right)$.

The point $p_{4}$, in the above theorem, is called the $\mathcal{C}$-orthocenter of the triangle $\triangle p_{1} p_{2} p_{3}$, and it is also evident that $p_{i}$ is the $\mathcal{C}$-orthocenter of the triangle $\triangle p_{j} p_{k} p_{l}$, where $\{i, j, k, l\}=\{1,2,3,4\}$. For these reason the set of four points $\left\{p_{1}, p_{2}, p_{3}, p_{4}\right\}$ is called a $\mathcal{C}$-orthocentric system. Then, it is clear that the set of the circumcenters $x_{l}$ of the triangles $\triangle p_{i} p_{j} p_{k}$ is a $\mathcal{C}$-orthocentric system too (see Theorem 2.9 in [8]). 
The notion of $\mathcal{C}$-orthocenter is define in strictly convex Minkowski plane because the strictly convex property guarantees that there exists only one circle passing through the three different points, i.e, each triangle with circumcenter has one and only one circumcenter. Note that the way Asplund and Grünbaum used to define the notion of $\mathcal{C}$-orthocenter needs three different circles of the circumcircle. However, there are cases where no three different circles exist of the circumcircle that holds the hypothesis of the previous theorem and therefore, in this situation we have a problem with this way to define the $\mathcal{C}$-orthocenter. Another problem with the definition of Asplund and Grübaum is that there are cases where the intersection of the three circles is more than one point and therefore, how to known which point is the $\mathcal{C}$-orthocenter of the triangle.

On the other hand, in Minkowski planes there are cases where a triangle can have many circumcenters or not have it and therefore, the same happen with the $\mathcal{C}$-orthocenters, but as our intention is to characterize the euclidianity in Minkowski planes, by studying some geometric properties of the $\mathcal{C}$-orthocentric systems, we will focus on defining the notion of $\mathcal{C}$-orthocenter for triangles having circumcenter. The problem here is how to decide which $\mathcal{C}$-orthocenter is associates with a particular circumcenter, when the triangle has many circumcenters.

Note that in the above theorem one can see that $x_{4}$ is the circumcenter of the triangle $\triangle p_{1} p_{2} p_{3}$ and the points $x_{1}, x_{2}, x_{3}$, centers of the circles, form a triangle where $p_{4}$ is its circumcenter. Furthermore, we can see that the triangles $\triangle p_{1} p_{2} p_{3}$ and $\triangle x_{1} x_{2} x_{3}$ are symmetric with respect to one point $q$ (see Figure 1). Following this ideas we will introduce in the section three a new way to define the notion of $\mathcal{C}$-orthocenter and $\mathcal{C}$-orthocentric systems.

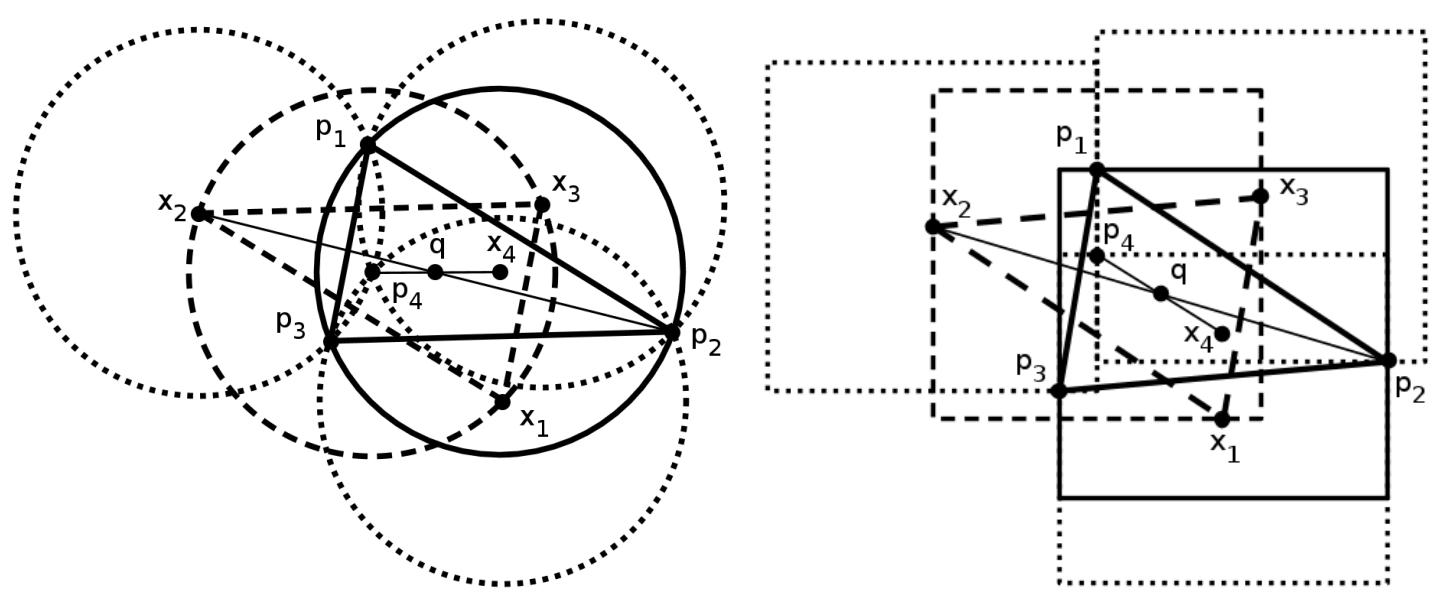

Figure 1: $\mathcal{C}$-orthocenter 


\section{Notations and Some Lemmas}

For our discussion, define $\mathcal{C}(\triangle a b c)=\{x \in M:\|x-a\|=\|x-b\|=\|x-c\|\}$ with $M$ a Minkowski plane, as the set of circumcenters of the triangle $\triangle a b c$. On the other hand, we denote by $H_{p,-2}$ and $S_{p}$, the homothety with center $p$ and ratio -2 and the the symmetry with center $p$ respectively, they are defined by $H_{p,-2}(w)=3 p-2 w$ and $S_{p}(w)=2 p-w$. Note that $S_{p}(w)-S_{p}(v)=v-w$. Thus, the symmetries are isometries in Minkowski planes, i.e., $\left\|S_{p}(w)-S_{p}(v)\right\|=\|w-v\|$ for all $w, v \in M$.

For $x \neq y$, we denote by $\langle x, y\rangle$ the line passing through $x$ and $y$, by $[x, y]$ the segment between $x$ and $y$, and by $[x, y\rangle$ the ray with starting point $x$ passing through $y$. For any two different points $x, y \in \mathcal{C}(w, \lambda)$ with $y \neq x$, the line $\langle x, y\rangle$ divides the plane into two half-planes, $L_{+}$and $L_{-}$, and therefore it divides $\mathcal{C}(w, \lambda)$ into two arcs between the points $x$ and $y, \mathcal{C}(w, \lambda) \cap L_{+}$and $\mathcal{C}(w, \lambda) \cap L_{-}$. They will be denote by $\operatorname{Arc}_{\mathcal{C}(w, \lambda)}^{+}(x, y)$ and $\operatorname{Arc}_{\mathcal{C}(w, \lambda)}^{-}(x, y)$. We need the definitions of Isosceles orthogonality, Birkhoff orthogonality, and Busemann angular bisectors. Let $x, y \in(M,\|\circ\|)$. The point $x$ is said to be isosceles orthogonal to $y$ if $\|x+y\|=\|x-y\|$, and in this case we write $x \perp_{I} y$ (cf. [2]). On the other hand, $x$ is said to be Birkhoff orthogonal to $y$ if $\|x+t y\| \geq\|x\|$ holds for all $t \in \mathbb{R}$, and for this we write $x \perp_{B} y$. We refer to [1] and [2] for basic properties of isosceles orthogonality and Birkhoff orthogonality, and the relations between them.

For non-collinear rays $[p, a\rangle$ and $[p, b\rangle$, the ray

$$
\left[p, \frac{1}{2}\left(\frac{a-p}{\|a-p\|}+\frac{b-p}{\|b-p\|}\right)+p\right\rangle
$$

is called the Busemann angular bisector of the angle spanned by $[p, a\rangle$ and $[p, b\rangle$, and it is denoted by $A_{B}([p, a\rangle,[p, b\rangle)$ (cf. [4], [5]). It is trivial to see that when $\|a-p\|=\|b-p\|$, then

$$
A_{B}([p, a\rangle,[p, b\rangle)=\left[p, \frac{a+b}{2}\right\rangle
$$

The followings lemmas are needed for our investigation.

Lemma 2.1. (cf. [9, (2.4)])If for any $x, y \in(M, \mathcal{C})$ with $x \perp_{I} y$ there exists a number $t>1$ such that $x \perp_{I}$ ty, then $(M, \mathcal{C})$ is Euclidean.

Lemma 2.2. (cf. [1, (10.9)]) A Minkowski plane $(M,\|\circ\|)$ is Euclidean if and only if the implication

$$
x \perp_{I} y \Longrightarrow x \perp_{B} y
$$

holds for all $x, y \in M$.

\section{Main Results}

In this section we present the main results, one of them will allow us to introduce the new way to define the notion of $\mathcal{C}$-orthocenter and therefore $\mathcal{C}$-orthocentric systems. 
The other will be used as support to show the results about characterizations of ecuclidean planes among normed planes, by studying geometric properties of $\mathcal{C}$-orthocentric systems.

Theorem 3.1. Let $M$ be a Minkowski plane. Let $x_{1}, x_{2}, x_{3}$ and $p_{4}$ be points in $M$. Let $m_{1}, m_{2}$ and $m_{3}$ be the midpoints of the segments $\left[x_{2}, x_{3}\right],\left[x_{1}, x_{3}\right]$ and $\left[x_{1}, x_{2}\right]$ respectively. Define the points $p_{i}=S_{m_{i}}\left(p_{4}\right)$, for $i=1,2,3$. Then the following holds:

1. The segments $\left[x_{i}, p_{i}\right]$ has the same midpoint $q$, for $i=1,2,3$. Also $2\left(q-m_{i}\right)=x_{i}-p_{4}$ for $i=1,2,3$, i.e,

$$
q=\frac{x_{1}+x_{2}+x_{3}-p_{4}}{2} .
$$

2. If $x_{4}=S_{q}\left(p_{4}\right)$, then $x_{i}-x_{j}=p_{j}-p_{i}$ for $\{i, j\} \subset\{1,2,3,4\}$.

3. $x_{i}-p_{j}=p_{k}-x_{l}$, where $\{i, j, k, l\}=\{1,2,3,4\}$.

4. If $g=\frac{x_{1}+x_{2}+x_{3}}{3}$, then $H_{g,-2}\left(p_{4}\right)=x_{4}$.

Proof. (1) Since $m_{i}=\frac{x_{j}+x_{k}}{2}$ for $\{i, j, k\}=\{1,2,3\}$ and

$$
p_{i}=S_{m_{i}}\left(p_{4}\right)=x_{j}+x_{k}-p_{4}
$$

then

$$
p_{i}+x_{i}=x_{i}+x_{j}+x_{k}-p_{4}
$$

for $\{i, j, k\}=\{1,2,3\}$. Thus,

$$
q=\frac{x_{1}+x_{2}+x_{3}-p_{4}}{2} .
$$

satisfied the propertie (see Figure 2).

(2) If $x_{4}=S_{q}\left(p_{4}\right)$, since $x_{i}=S_{q}\left(p_{i}\right)$ for $i=1,2,3$, then

$$
x_{i}-x_{j}=S_{q}\left(p_{i}\right)-S_{q}\left(p_{j}\right)=p_{j}-p_{i}
$$

for $\{i, j\} \subset\{1,2,3,4\}$

(3) Let $d_{i}=S_{q}\left(m_{i}\right)$ for $i=1,2,3$, then $d_{i}$ are the midpoints of the segments $\left[p_{j}, p_{k}\right]$ for $\{i, j, k\}=\{1,2,3\}$. Then, using the equation (3.1) and the simmetry $S_{q}$ we have

$$
x_{i}=p_{j}+p_{k}-x_{4}
$$

for $\{i, j, k\}=\{1,2,3\}$. From equations (3.3) and (3.4), then we get the propiertie desired.

(4) Since $g=\frac{x_{1}+x_{2}+x_{3}}{3}$ and by the homothety definition, then

$$
H_{g,-2}\left(p_{4}\right)=3 g-2 p_{4}=3\left(\frac{x_{1}+x_{2}+x_{3}}{3}\right)-2 p_{4}=x_{4}
$$




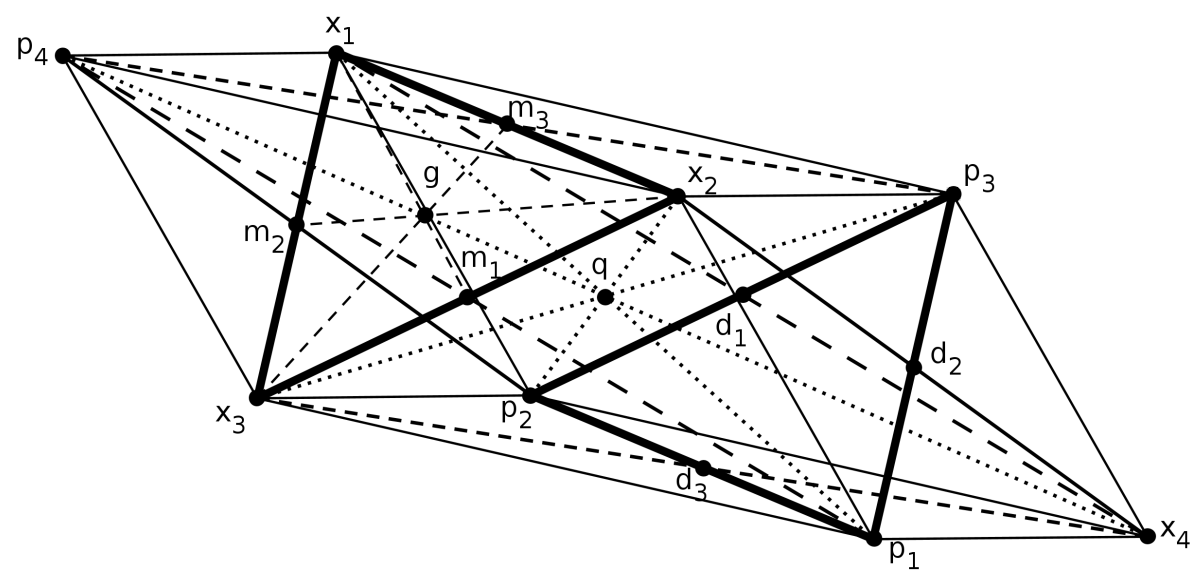

Figure 2: Demonstration Theorem 3.1

By the construction shown in Theorem (3.1), we will call $p_{4}$-Antitriangle of the triangle $\triangle x_{1} x_{2} x_{3}$ to the triangle $\triangle p_{1} p_{2} p_{3}$, i.e, the Antitriangle with respect to the point $p_{4}$. Now, we can introduce the new way to define the notion of $\mathcal{C}$-orthocenter saying: given the points $x_{1}, x_{2}, x_{3}$, and $p_{4}$ in $\mathcal{C}\left(\triangle x_{1} x_{2} x_{3}\right)$, we will say that $x_{4}$ is the $\mathcal{C}$-orthocenter of the triangle $\triangle x_{1} x_{2} x_{3}$ associated with $p_{4}$ if $S_{q}\left(p_{4}\right)=x_{4}$, where $q$ is the point of symmetry of the triangle $\triangle x_{1} x_{2} x_{3}$ and its $p_{4}$-Antitriangle as we defined in the Theorem (3.1). Furthermore, if we have four points $p_{1}, p_{2}, p_{3}$ and $p_{4}$ in the plane. We will say that $\left\{p_{1}, p_{2}, p_{3}, p_{4}\right\}$ is a $\mathcal{C}$-orthocentric system if there exist a circumcenter $x_{i}$ of the triangle $\triangle p_{j} p_{k} p_{l}$, with $\{i, j, k, l\}=\{1,2,3,4\}$ such that $p_{i}$ is the $\mathcal{C}$-orthocenter of the triangle $\triangle p_{j} p_{k} p_{l}$. Then, let $\mathcal{H}(\triangle a b c)=\{x \in M: x$ is a $\mathcal{C}$-orthocenter of the triangle $\}$ be the set of $\mathcal{C}$-orthocenters of the triangle $\triangle a b c$.

As we said at the beginning, $x_{4}$ is the circumcenter of the $p_{4}$-Antitriangle of the triangle $\triangle x_{1} x_{2} x_{3}$ and it holds the definition given by E. Asplund and B. Grünbaum in [3]. In this way one can see the relation between the $\mathcal{C}$-orthocenters and the circumcenters of the triangle $\triangle x_{1} x_{2} x_{3}$ and we show the uniqueness of the $\mathcal{C}$-orthocenter, given the circumcenter of the triangle.

Corollary 3.2. With the assumptions of the previous theorem. Then the following holds:

1. If $x_{4}=S_{q}\left(p_{4}\right)$, then $x_{4}=x_{1}+x_{2}+x_{3}-2 p_{4}$

2. $m_{i}=\frac{p_{i}+p_{4}}{2}$ for all $i=1,2,3$.

3. If $g=\frac{x_{1}+x_{2}+x_{3}}{3}$ and $g_{1}=\frac{p_{1}+p_{2}+p_{3}}{3}$, then $g_{1}=S_{q}(g)$ and $S_{g}\left(p_{4}\right)=g_{1}$.

Proof. (1) By Theorem (3.1) $q=\frac{x_{1}+x_{2}+x_{3}-p_{4}}{2}$ and, since $q=\frac{x_{4}+p_{4}}{2}$, then $x_{4}=x_{1}+x_{2}+x_{3}-2 p_{4}$.

(2) Since $m_{i}=\frac{x_{j}+x_{k}}{2}$ for $\{i, j, k\}=\{1,2,3\}$, then by (3) from Theorem 3.5) (ver Figura 3), we get

$$
m_{i}=\frac{x_{j}+x_{k}}{2}=\frac{p_{i}+p_{l}}{2}
$$


for $\{i, j, k, l\}=\{1,2,3,4\}$.

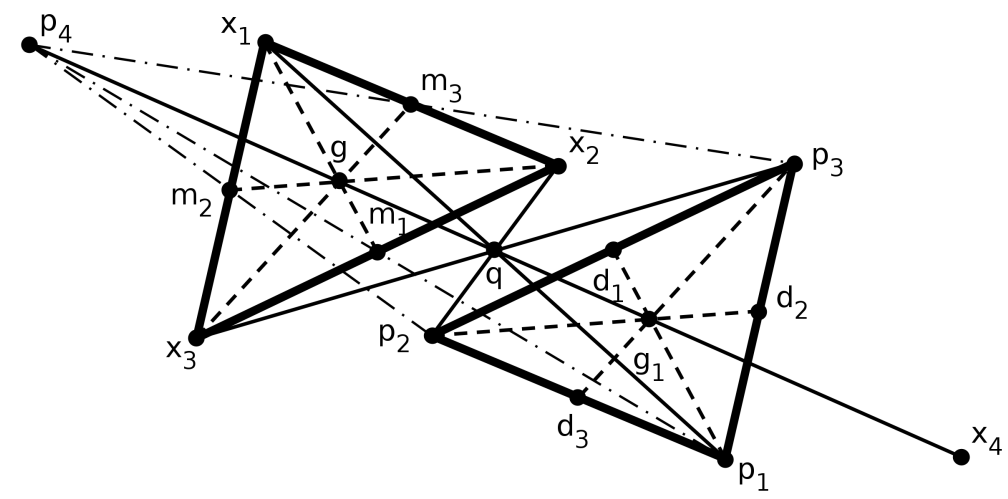

Figure 3: Demonstration Corollary 3.2

(3) Since $p_{i}=S_{m_{i}}\left(p_{4}\right)$ for $i=1,2,3$ (see Figure 3), then

$$
g_{1}=\frac{p_{1}+p_{2}+p_{3}}{3}=\frac{2 x_{1}+2 x_{2}+2 x_{3}-3 p_{4}}{3}=2 g-p_{4}=S_{g}\left(p_{4}\right)
$$

On the other hand, since $g_{1}=\frac{p_{1}+p_{2}+p_{3}}{3}$,

$$
g_{1}=\frac{2\left(m_{1}+m_{2}+m_{3}\right)-3 p_{4}}{3}=x_{1}+x_{2}+x_{3}-p_{4}-\frac{x_{1}+x_{2}+x_{3}}{3}=S_{q}(g)
$$

Corollary 3.3. With the assumptions of the previous theorem. Let $p_{4}$ in $\mathcal{C}\left(\triangle x_{1} x_{2} x_{3}\right)$, and $d_{1}, d_{2}$ and $d_{3}$ be the midpoints sides of the $p_{4}$-Antitriangle of the triangle $\triangle x_{1} x_{2} x_{3}$ respectively, then the following holds:

1. $\left\{x_{1}, x_{2}, x_{3}, x_{4}\right\}$ and $\left\{p_{1}, p_{2}, p_{3}, p_{4}\right\}$ are $\mathcal{C}$-orthocentrics systems.

2. The points $d_{1}, d_{2}$ and $d_{3}$ are the midpoints of the segments that join the vertices of the triangle $\triangle x_{1} x_{2} x_{3}$ with its $\mathcal{C}$-orthocenter.

3. The midpoints sides of the triangle $\triangle x_{1} x_{2} x_{3}$ and its $p_{4}$-Antitriangle, lies in the circle with center $q$ and radio $\left\|q-m_{1}\right\|$ (The Six Points Circle).

Proof. (1) Since $p_{4}$ is the circumcenter of the triangle $\triangle x_{1} x_{2} x_{3}$ and by the construction used in the Theorem (3.1) we get that $x_{4}=S_{q}\left(p_{4}\right)$, so $x_{4}$ is the $\mathcal{C}$-orthocenter of the triangle $\triangle x_{1} x_{2} x_{3}$. Furthermore, since $p_{i}$ is the circumcenter of the triangle $\triangle x_{j} x_{k} x_{j}$ for $\{i, j, k, l\}=\{1,2,3,4\}$, and $x_{i}=S_{q}\left(p_{i}\right)$ for $i=1,2,3$, then the sets $\left\{x_{1}, x_{2}, x_{3}, x_{4}\right\}$ and $\left\{p_{1}, p_{2}, p_{3}, p_{4}\right\}$ are $\mathcal{C}$-orthocentric systems (see Figure 4 ).

(2) Since $d_{i}=\frac{p_{j}+p_{k}}{2}$ for $\{i, j, k\}=\{1,2,3\}$ and $p_{i}=S_{m_{i}}\left(p_{4}\right)$, then

$$
d_{i}=\frac{p_{j}+p_{k}}{2}=\frac{2 m_{j}-p_{4}+2 m_{k}-p_{4}}{2}=\frac{2 x_{i}+x_{k}+x_{j}-2 p_{4}}{2}=\frac{x_{i}+x_{4}}{2}
$$




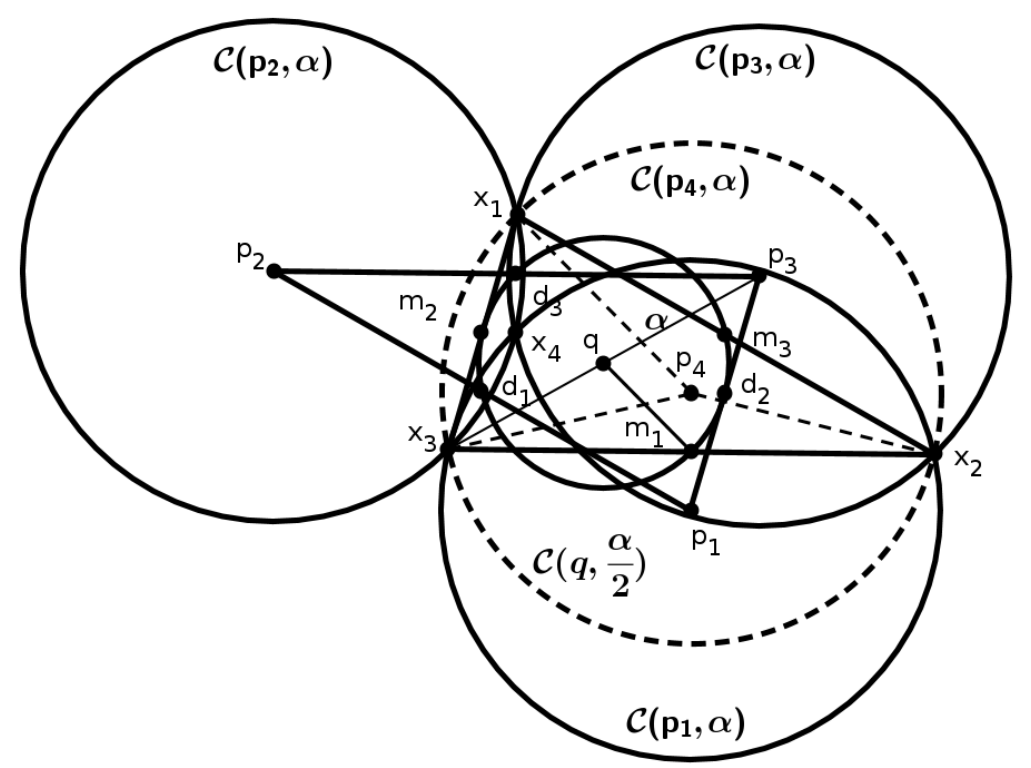

Figure 4: Six Points Circle

for $\{i, j, k\}=\{1,2,3\}$.

(3) Since $p_{4}$ is the circumcenter of the triangle $\triangle x_{1} x_{2} x_{3}$, then

$$
\left\|p_{4}-x_{i}\right\|=\alpha
$$

for $i=1,2,3$. Now, to prove that $\mathcal{C}\left(q,\left\|q-m_{1}\right\|\right)$ passes through the midpoints side of the triangles $\triangle x_{1} x_{2} x_{3}$ and $\triangle p_{1} p_{2} p_{3}$, is enough to see that

$$
\left\|q-m_{i}\right\|=\alpha \quad \text { y } \quad\left\|q-d_{i}\right\|=\alpha
$$

for $i=1,2,3$. Then, notice that

$$
\left\|q-m_{i}\right\|=\frac{\left\|p_{4}-x_{i}\right\|}{2}
$$

for $i=1,2,3$.

On the other hand, $d_{i}=2 q-m_{i}$ for $i=1,2,3$ and therefore

$$
\left\|q-d_{i}\right\|=\left\|q-m_{i}\right\|
$$

for $i=1,2,3$. Then, by the equations (3.5) and (3.6) the points $m_{1}, m_{2}, m_{3}, d_{1}, d_{2}$ and $d_{3}$ lie in the circle $\mathcal{C}\left(q, \frac{\alpha}{2}\right)$ (see Figure 4 ).

In Euclidean Geometry is well known the alinement relation between the barycenter, the circumcenter and the orthocenter of a triangle. The line passing through these points is called Euler's Line of the triangle. If " $o$ " is the circumcenter, " $g$ " the barycenter and 
" $h$ " the orthocenter of a triangle, it is well known that " $g$ " is an interior point of the segment $[o, h]$, and if the three points are different, then the ratio $\frac{h g}{o g}=-2$. Also these properties holds in strictly convex Minkowski plane. The following corollary shows the generalization of the notion of Euler's line in Minkowski planes using the structure of $\mathcal{C}$-orthocenter, and show that " $h$ " is the image of " $o$ " in the homothety with center " $g$ " and ratio -2 . So, the Euler's Line Theorem in Minkowski planes would be expressed as follows:

Corollary 3.4. Let $M$ a Minkowski plane and $x_{1}, x_{2}, x_{3}$ points of $M$. If $g=\frac{x_{1}+x_{2}+x_{3}}{3}$, the barycenter of the triangle $\triangle x_{1} x_{2} x_{3}$, then $H_{g,-2}\left(\mathcal{C}\left(\triangle x_{1} x_{2} x_{3}\right)\right)=\mathcal{H}\left(\triangle x_{1} x_{2} x_{3}\right)$.

Proof. Take $p_{4} \in \mathcal{C}\left(\triangle x_{1} x_{2} x_{3}\right)$, then by (1) from Corollary (3.3) there exists a point $x_{4} \in \mathcal{H}\left(\triangle x_{1} x_{2} x_{3}\right)$ such that $\left\{x_{1}, x_{2}, x_{3}, x_{4}\right\}$ is a $\mathcal{C}$-orthocentric system. Then, by (4) from Theorem (3.1) $H_{g,-2}\left(p_{4}\right)=x_{4}$ and therefore $H_{g,-2}\left(\mathcal{C}\left(\triangle x_{1} x_{2} x_{3}\right)\right) \subset \mathcal{H}\left(\triangle x_{1} x_{2} x_{3}\right)$.

Conversely let $x_{4} \in \mathcal{H}\left(\triangle x_{1} x_{2} x_{3}\right)$, then there exists $p_{4} \in \mathcal{C}\left(\triangle x_{1} x_{2} x_{3}\right)$ such that $S_{q}\left(p_{4}\right)=x_{4}$, with $q$ the point of symmetry of the triangles $\triangle x_{1} x_{2} x_{3}$ and its $p_{4}$-Antitriangle. Then, $x_{4}$ is the circumcenter of the $p_{4}$-Antitriangle. Thus, $p_{4}$ holds that $H_{g,-2}\left(p_{4}\right)=x_{4}$ and therefore $\mathcal{H}\left(\triangle x_{1} x_{2} x_{3}\right) \subset H_{g,-2}\left(\mathcal{C}\left(\triangle x_{1} x_{2} x_{3}\right)\right)$.

The following Lemma will give us the tools to proof the results about characterizations of euclidean planes by studying geometric properties of $\mathcal{C}$-orthocentric systems in normed planes.

Lemma 3.5. Let $(M,\|\circ\|)$ be a Minkowski plane, with origen $O$. For any $x, z \in M$ with $x \perp_{I} z$, let $p_{3}=-z, p_{4}=z, x_{1}=x, x_{2}=-x$, and $\lambda=\|x+z\|$. Then there are points $x_{3} \in \mathcal{C}\left(p_{4}, \lambda\right)$ and $q \in \mathcal{C}(O, \lambda / 2)$, such that $\left\{p_{1}, p_{2}, p_{3}, p_{4}\right\}$ and $\left\{x_{1}, x_{2}, x_{3}, x_{4}\right\}$ are $\mathcal{C}$-orthocentric systems, where $p_{1}=S_{q}\left(x_{1}\right), p_{2}=S_{q}\left(x_{2}\right)$ and $x_{4}=S_{q}\left(p_{4}\right)$. Furthermore, if $L_{1}=\left\langle S_{x_{1}}\left(p_{3}\right), S_{x_{2}}\left(p_{3}\right)\right\rangle$ and $L_{3}$ is the parallel line to $L_{1}$ passing through $p_{4}$, then the following statements holds:

1. $p_{1} \in \mathcal{C}\left(x_{2}, \lambda\right), p_{2} \in \mathcal{C}\left(x_{1}, \lambda\right)$ and $x_{4} \in \mathcal{C}\left(p_{3}, \lambda\right)$

2. There are points $p_{1}$ and $p_{2}$ such that $\left\|p_{3}-p_{1}\right\|=\left\|p_{3}-p_{2}\right\|$.

3. $p_{1}= \pm \frac{\lambda z}{\|z\|}-x$ and $p_{2}= \pm \frac{\lambda z}{\|z\|}+x$ if and only if $p_{4} \in\left\langle p_{3}, \frac{p_{1}+p_{2}}{2}\right\rangle$. Particularly, $p_{1}=\frac{\lambda z}{\|z\|}-x$ and $p_{2}=\frac{\lambda z}{\|z\|}+x$ if and only if $p_{4} \in\left[p_{3}, \frac{p_{1}+p_{2}}{2}\right]$.

4. If $\|z\|<\lambda$ and $q \in \operatorname{Arc}_{\mathcal{C}\left(O, \frac{\lambda}{2}\right)}^{-}\left(\frac{p_{4}+x_{2}}{2}, \frac{p_{4}+x_{1}}{2}\right)$, with $p_{1} \neq p_{4}, S_{x_{2}}\left(p_{3}\right)$, then $p_{3}$ and the line $\left\langle p_{1}, p_{2}\right\rangle$ are separated by $L_{1}$.

5. There are points $p_{1}$ and $p_{2}$ such that the line $\left\langle p_{1}, p_{2}\right\rangle$ and $p_{3}$ are separated by $L_{1}$, or $\left\langle p_{1}, p_{2}\right\rangle=L_{1}$. Furthermore $p_{4} \in A_{B}\left(\left[p_{3}, p_{1}\right\rangle,\left[p_{3}, p_{2}\right\rangle\right)$.

Proof. Takes the circle $\mathcal{C}\left(p_{4}, \lambda\right)$. If $x_{3} \in \mathcal{C}\left(p_{4}, \lambda\right)$, then $p_{4}$ is the cincumcenter of the triangle $\triangle x_{1} x_{2} x_{3}$. By (1) from Theorem $(3.1), q=\frac{p_{3}+x_{3}}{2}$ and therefore $p_{1}=S_{q}\left(x_{1}\right)$, $p_{2}=S_{q}\left(x_{2}\right)$ and $x_{4}=S_{q}\left(p_{4}\right)$. So, by (1) from Corollary (3.3), the sets $\left\{p_{1}, p_{2}, p_{3}, p_{3}\right\}$ and $\left\{x_{1}, x_{2}, x_{3}, x_{4}\right\}$ are $\mathcal{C}$-orthocentric systems. 


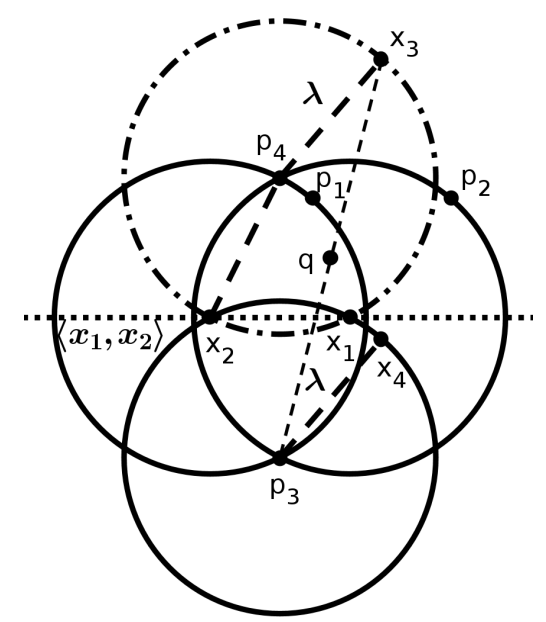

Figure 5: Circles $\mathcal{C}\left(x_{4}, \lambda\right)$ and $\mathcal{C}\left(p_{4}, \lambda\right)$

(1) To prove that $p_{1} \in \mathcal{C}\left(x_{2}, \lambda\right), p_{2} \in \mathcal{C}\left(x_{1}, \lambda\right)$ and $x_{4} \in \mathcal{C}\left(p_{3}, \lambda\right)$ we have to see that

$$
\left\|x_{1}-p_{2}\right\|=\left\|x_{2}-p_{1}\right\|=\left\|p_{3}-x_{4}\right\|=\lambda
$$

Note that $\left\|p_{4}-x_{3}\right\|=\lambda$, then $\lambda=2\|q\|$ so that

$$
\|q\|=\frac{\lambda}{2}
$$

Since $p_{2}=S_{q}\left(x_{2}\right), p_{1}=S_{q}\left(x_{1}\right), x_{4}=S_{q}\left(p_{4}\right)$ (see Figure 5), and ussing (3.7), then

$$
\left\|x_{1}-p_{2}\right\|=\left\|x_{2}-p_{1}\right\|=\left\|p_{3}-x_{4}\right\|=2\|q\|=\lambda
$$

(2) Without loss of generality takes the $\operatorname{arc} \operatorname{Arc}_{\mathcal{C}\left(p_{4}, \lambda\right)}^{+}\left(x_{1}, x_{2}\right)$, which is a closed set (see Figure 55. Define the continuous parameterization $\alpha:[0,1] \longrightarrow \operatorname{Arc}_{\mathcal{C}\left(p_{4}, \lambda\right)}^{+}\left(x_{1}, x_{2}\right)$, such that

$$
\lim _{t \rightarrow 0} \alpha(t)=x_{1} \quad \lim _{t \rightarrow 1} \alpha(t)=x_{2}
$$

Define the continuous function $f: \operatorname{Arc}_{\mathcal{C}\left(p_{4}, \lambda\right)}^{+}\left(x_{1}, x_{2}\right) \longrightarrow \mathbb{R}$ by:

$$
f(x)=\left\|x_{2}-x\right\|-\left\|x_{1}-x\right\|
$$

On the other hand, for any $x \in \operatorname{Arc}_{\mathcal{C}\left(p_{4}, \lambda\right)}^{+}\left(x_{1}, x_{2}\right)$ there exists $t \in[0,1]$ such that $\alpha(t)=x$, then by (3.8) and (3.9) $f(\alpha(t))$ holds:

$$
\lim _{t \rightarrow 0} f(\alpha(t))=\left\|x_{2}-x_{1}\right\|>0 \quad \text { and } \quad \lim _{t \rightarrow 1} f(\alpha(t))=-\left\|x_{1}-x_{2}\right\|<0
$$

Then, since $f$ is defined on a compact set, taking negative and positive values, there exists $x_{3} \in \operatorname{Arc}_{\mathcal{C}\left(p_{4}, \lambda\right)}^{+}\left(x_{1}, x_{2}\right)$ such that $f\left(x_{3}\right)=0$, i.e, a $x_{3}$ that holds

$$
\left\|x_{1}-x_{3}\right\|=\left\|x_{2}-x_{3}\right\|
$$




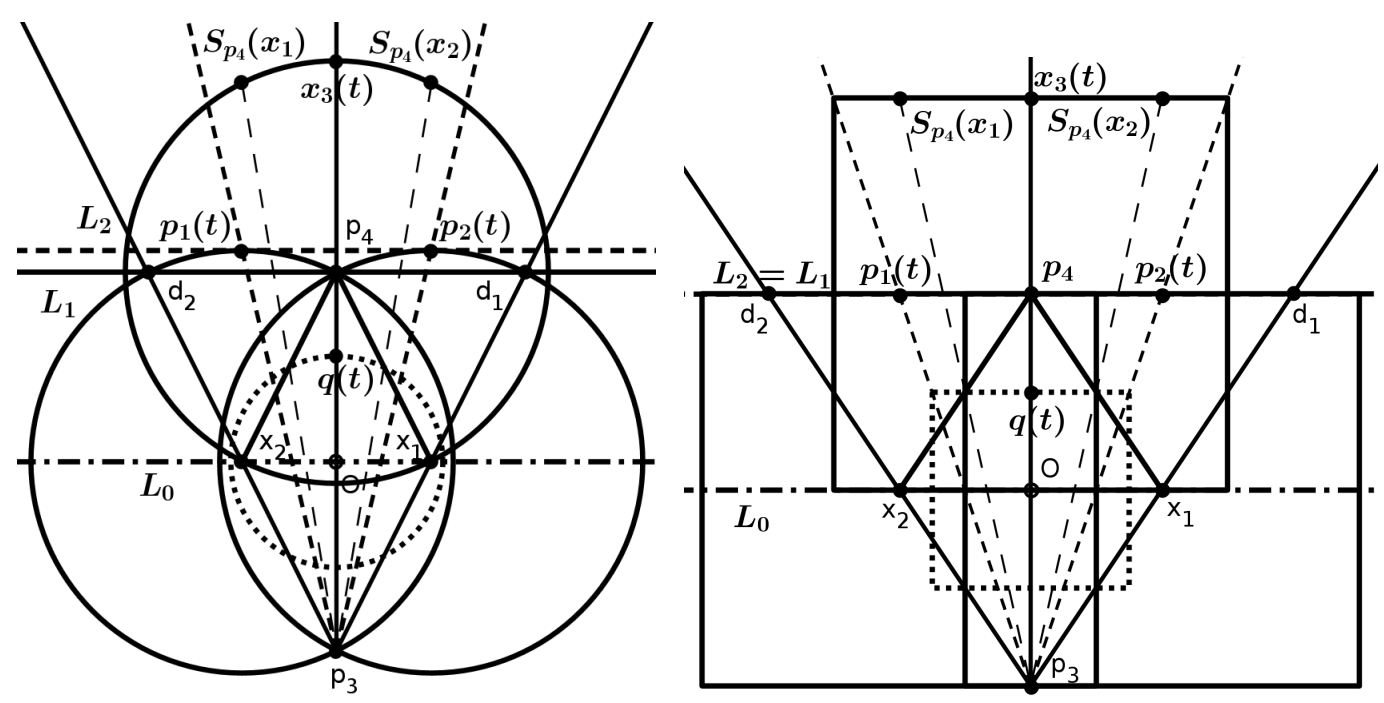

Figure 6: Demonstration (3) and (5), Lemma 3.5

Now, define the points $q=\frac{x_{3}+p_{3}}{2}, p_{1}=S_{q}\left(x_{1}\right)$ and $p_{2}=S_{q}\left(x_{2}\right)$. By 3.10$)$, and the fact that $S_{q}(x)$ is a isometry, then $\left\|p_{3}-p_{1}\right\|=\left\|p_{3}-p_{2}\right\|$.

(3) To get that $p_{4} \in\left\langle p_{3}, \frac{p_{1}+p_{2}}{2}\right\rangle$, since $p_{4}=z$ and $p_{3}=-z, \frac{p_{1}+p_{2}}{2}$ need to be of the form $k z$, with $k \in \mathbb{R}$ (see Figure 6). Since $\frac{p_{1}+p_{2}}{2}=2 q$, then $q=\frac{k z}{2}$ and by the equation (3.7)

$$
\frac{\lambda}{2}=\|q\|=\left\|\frac{k z}{2}\right\|
$$

therefore $k= \pm \frac{\lambda}{\|z\|}$. So, $q= \pm \frac{\lambda z}{2\|z\|}$ are the only possible values for which the condition is satisfied. Then, the only pair of points $\left\{p_{1}, p_{2}\right\}$ would be $\left\{\frac{\lambda z}{\|z\|}-x, \frac{\lambda z}{\|z\|}+x\right\}$ and $\left\{-\frac{\lambda z}{\|z\|}-x,-\frac{\lambda z}{\|z\|}+x\right\}$.

(4) Since $p_{1} \notin\left\{p_{4}, S_{x_{2}}\left(p_{3}\right)\right\},\|z\|<\lambda$, and by the equation (3.7), we have that $p_{4} \neq \frac{p_{1}+p_{2}}{2}=2 q$. Furthermore, the lines $\left\langle p_{1}, p_{2}\right\rangle$ and $\left\langle S_{x_{1}}\left(p_{3}\right), S_{x_{2}}\left(p_{3}\right)\right\rangle$ are different and they are paralles because

$$
S_{x_{1}}\left(p_{3}\right)-S_{x_{2}}\left(p_{3}\right)=2\left(p_{2}-p_{1}\right)
$$

Now, if $q \in \operatorname{Arc}_{\mathcal{C}\left(O, \frac{\lambda}{2}\right)}^{-}\left(\frac{p_{4}+x_{2}}{2}, \frac{p_{4}+x_{1}}{2}\right)$, then $p_{1}$ lies in the $\operatorname{Arc}_{\mathcal{C}\left(x_{2}, \lambda\right)}^{-}\left(S_{x_{2}}\left(p_{3}\right), p_{4}\right)$. and $p_{2}$ lies in the $\operatorname{Arc}_{\mathcal{C}\left(x_{1}, \lambda\right)}^{-}\left(p_{4}, S_{x_{1}}\left(p_{3}\right)\right)$ (see Figure 7 . Also, $\frac{p_{1}+p_{2}}{2}$ and $p_{3}$ are separated by $L_{1}=\left\langle S_{x_{2}}\left(p_{3}\right), S_{x_{1}}\left(p_{3}\right)\right\rangle$. Since $\frac{p_{1}+p_{2}}{2} \in\left[p_{1}, p_{2}\right]$ and the equation 3.11 , then $\left\langle p_{1}, p_{2}\right\rangle$ and $p_{3}$ are separated by $L_{1}$.

(5) For any $t \in(0,1)$, define the continuous functions

$$
x_{3}(t):[0,1] \longrightarrow \operatorname{Arc}_{\mathcal{C}\left(p_{4}, \lambda\right)}^{+}\left(S_{p_{4}}\left(x_{1}\right), S_{p_{4}}\left(x_{2}\right)\right)
$$




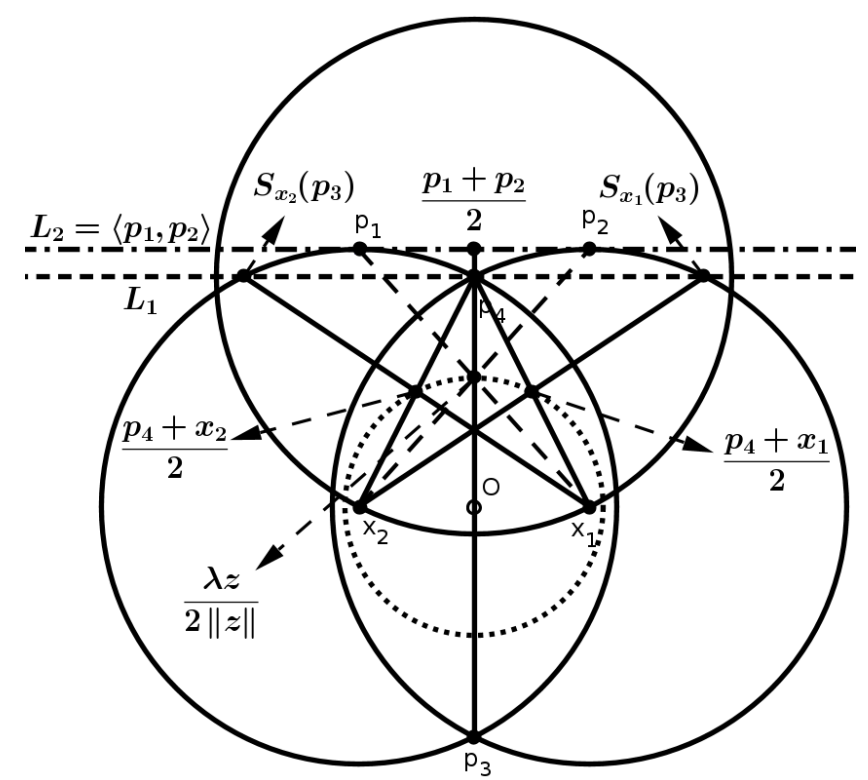

Figure 7: Lines $\left\langle p_{1}, p_{2}\right\rangle$ and $\left\langle S_{x_{1}}\left(p_{3}\right), S_{x_{2}}\left(p_{3}\right)\right\rangle$

$q(t)=\frac{p_{3}+x_{3}(t)}{2}$ and $p_{i}(t)=2 q(t)-x_{i}$ for $i=1,2$. When $t$ is moving from 0 to 1 , the ray $A_{B}\left(\left[p_{3}, p_{1}(t)\right\rangle,\left[p_{3}, p_{2}(t)\right\rangle\right)$ continually goes from $A_{B}\left(\left[p_{3}, d_{2}\right\rangle,\left[p_{3}, p_{4}\right\rangle\right)$ to $A_{B}\left(\left[p_{3}, p_{4}\right\rangle,\left[p_{3}, d_{1}\right\rangle\right)$ (see Figure 6). So, there exists $t_{0} \in(0,1)$ such that

$$
A_{B}\left(\left[p_{3}, p_{1}\left(t_{0}\right)\right\rangle,\left[p_{3}, p_{2}\left(t_{0}\right)\right\rangle\right)=\left[p_{3}, p_{4}\right\rangle
$$

Now, let $p_{1}=p_{1}\left(t_{0}\right)$ and $p_{2}=p_{2}\left(t_{0}\right)$, then $p_{1}$ and $p_{2}$ have the desired property. By (4) from this Lemma, $p_{3}$ and the line $\left\langle p_{1}, p_{2}\right\rangle$ are separated by the line $L_{1}$ passing through $p_{4}$ parallel to $\left\langle p_{1}, p_{2}\right\rangle$, or $\left\langle p_{1}, p_{2}\right\rangle=L_{1}$.

Theorem 3.6. A Minkowski plane $(M,\|\circ\|)$ is euclidean if and only if for any $\mathcal{C}$-orthocentric system $\left\{p_{1}, p_{2}, p_{3}, p_{4}\right\}$ the relation

$$
\left(p_{i}-p_{j}\right) \perp_{B}\left(p_{k}-p_{l}\right)
$$

holds, where $\{i, j, k, l\}=\{1,2,3,4\}$

Proof. If $(M,\|\circ\|)$ is euclidean, then for any $\mathcal{C}$-orthocentric system $\left\{p_{1}, p_{2}, p_{3}, p_{4}\right\}, p_{i}$ is the $\mathcal{C}$-orthocenter of $\triangle p_{j} p_{k} p_{l}$ for $\{i, j, k, l\}=\{1,2,3,4\}$. Furthermore

$$
\left(p_{i}-p_{j}\right) \perp_{B}\left(p_{k}-p_{l}\right)
$$

Conversely, for any $x, y \in M$ with $x \perp_{I} y$, let

$$
p_{4}=y \quad p_{3}=-y \quad x_{1}=x \quad x_{2}=-x
$$


By Lemma 3.5, there are two points $p_{1}$ and $p_{2}$ such that $\left\{p_{1}, p_{2}, p_{3}, p_{4}\right\}$ is a $\mathcal{C}$-orthocentric system (see Figure 5). By Theorem (3.1) we have

$$
p_{2}-p_{1}=x_{1}-x_{2}=2 x
$$

Since $\left(p_{2}-p_{1}\right) \perp_{B}\left(p_{4}-p_{3}\right)$ and $p_{4}-p_{3}=2 y$, then $x \perp_{B} y$. So, by Lemma 2.2$),(M,\|\circ\|)$ is euclidean.

Theorem 3.7. A Minkowski plane $(M,\|\circ\|)$ is euclidean if and only if for any $\mathcal{C}$-orthocentric system $\left\{p_{1}, p_{2}, p_{3}, p_{4}\right\}$, with $\left\|p_{3}-p_{1}\right\|=\left\|p_{3}-p_{2}\right\|$, it holds that $p_{4} \in\left\langle p_{3}, \frac{p_{1}+p_{2}}{2}\right\rangle$.

Proof. If $(M,\|\circ\|)$ is euclidean, any $\mathcal{C}$-orthocentric system holds that $p_{4} \in\left\langle p_{3}, \frac{p_{1}+p_{2}}{2}\right\rangle$ if $\left\|p_{3}-p_{1}\right\|=\left\|p_{3}-p_{2}\right\|$.

Conversely, let $x, y \in(M,\|\circ\|)$ such that $x \perp_{I} y$, by Lemma (2.1) is enough to prove that there exists a $t>1$ such that $x \perp_{I} t y$. Clearly it exists such $t$ if $x=O$ or $y=O$. Now, let $x, y \in M \backslash\{O\}$ with $x \perp_{I} y$. Define $\lambda=\|x+y\|$ and

$$
p_{4}=y \quad p_{3}=-y \quad x_{1}=x \quad x_{2}=-x
$$

then by (2) from Lemma (3.5) we have points $p_{1}$ and $p_{2}$ such that

$$
\left\|p_{3}-p_{1}\right\|=\left\|p_{3}-p_{2}\right\|
$$

By hypothesis $p_{4} \in\left\langle p_{3}, \frac{p_{1}+p_{2}}{2}\right\rangle$, and ussing (3) from Lemma 3.5 , we can take $p_{1}=$ $\frac{\lambda y}{\|y\|}-x$ and $p_{2}=\frac{\lambda y}{\|y\|}+x$. So,

$$
\begin{aligned}
& \left\|p_{3}-p_{1}\right\|=\left\|x-\left(1+\frac{\lambda}{\|y\|}\right) y\right\| \\
& \left\|p_{3}-p_{2}\right\|=\left\|x+\left(1+\frac{\lambda}{\|y\|}\right) y\right\|
\end{aligned}
$$

and by the equation 3.12 , then $x \perp_{I} t y$ where $t=1+\frac{\lambda}{\|y\|}>1$, since $\lambda>0$.

Theorem 3.8. A Minkowski plane $(M,\|\circ\|)$ is euclidean if and only if for any $\mathcal{C}$-orthocentric system $\left\{p_{1}, p_{2}, p_{3}, p_{4}\right\}$, the equality $\left\|p_{3}-p_{1}\right\|=\left\|p_{3}-p_{2}\right\|$ holds, whenever $p_{4} \in\left\langle p_{3}, \frac{p_{1}+p_{2}}{2}\right\rangle$.

Proof. If $(M,\|\circ\|)$ is euclidean, any $\mathcal{C}$-orthocentric system, with $p_{4} \in\left\langle p_{3}, \frac{p_{1}+p_{2}}{2}\right\rangle$, holds that $\left\|p_{3}-p_{1}\right\|=\left\|p_{3}-p_{2}\right\|$.

Conversely, let $x, y \in(M,\|\circ\|)$ with $x \perp_{I} y$, by Lemma (2.1) is enough to prove that there exists $t>1$ such that $x \perp_{I} t y$. Clearly it exists such $t$ if $x=O$ or $y=O$. Now, let $x, y \in M \backslash\{O\}$ with $x \perp_{I} y$. Define $\lambda=\|x+y\|$ and

$$
p_{4}=y \quad p_{3}=-y \quad x_{1}=x \quad x_{2}=-x
$$

then by (3) from Lemma 3.5 we have the points $p_{1}= \pm \frac{\lambda y}{\|y\|}-x$ and $p_{2}= \pm \frac{\lambda y}{\|y\|}+x$. Take the values $p_{1}=\frac{\lambda y}{\|y\|}-x$ and $p_{2}=\frac{\lambda y}{\|y\|}+x$, and since $\left\|p_{3}-p_{1}\right\|=\left\|p_{3}-p_{2}\right\|$, then $x \perp_{I}$ ty with $t=1+\frac{\lambda}{\|y\|}>1$. 
Theorem 3.9. A Minkowski plane $(M,\|\circ\|)$ is euclidean if and only if for any $\mathcal{C}$-orthocentric system $\left\{p_{1}, p_{2}, p_{3}, p_{4}\right\}, p_{4}$ lies on the line containing $A_{B}\left(\left[p_{3}, p_{1}\right\rangle,\left[p_{3}, p_{2}\right\rangle\right)$ whenever $\left\|p_{3}-p_{1}\right\|=\left\|p_{3}-p_{2}\right\|$.

Proof. If $(M,\|\circ\|)$ is euclidean, any $\mathcal{C}$-orthocentric system with $\left\|p_{3}-p_{1}\right\|=\left\|p_{3}-p_{2}\right\|$, holds that $p_{4}$ lies on the line containing $A_{B}\left(\left[p_{3}, p_{1}\right\rangle,\left[p_{3}, p_{2}\right\rangle\right)$.

Conversely, let $x, y \in(M,\|\circ\|)$ with $x \perp_{I} y$. By Theorem (3.7), we only have to proof that for any $\mathcal{C}$-orthocentric system $\left\{p_{1}, p_{2}, p_{3}, p_{4}\right\}, p_{4} \in\left\langle p_{3}, \frac{p_{1}+p_{2}}{2}\right\rangle$ whenever $\left\|p_{3}-p_{1}\right\|=\left\|p_{3}-p_{2}\right\|$. By the definition of Busemann angular bisectors and the fact that $\left\|p_{3}-p_{1}\right\|=\left\|p_{3}-p_{2}\right\|$, then

$$
A_{B}\left(\left[p_{3}, p_{1}\right\rangle,\left[p_{3}, p_{2}\right\rangle\right)=\left[p_{3}, \frac{p_{1}+p_{2}}{2}\right\rangle
$$

Thus $\left\langle p_{3}, \frac{p_{1}+p_{2}}{2}\right\rangle$ is the line containing $A_{B}\left(\left[p_{3}, p_{1}\right\rangle\left[p_{3}, p_{2}\right\rangle\right)$ and therefore $p_{4} \in\left\langle p_{3}, \frac{p_{1}+p_{2}}{2}\right\rangle$.

Theorem 3.10. A Minkowski plane $(M,\|\circ\|)$ is euclidean if and only if for any $\mathcal{C}$-orthocentric system $\left\{p_{1}, p_{2}, p_{3}, p_{4}\right\}$, the equality $\left\|p_{3}-p_{1}\right\|=\left\|p_{3}-p_{2}\right\|$ holds whenever $p_{4}$ lies on the line containing $A_{B}\left(\left[p_{3}, p_{1}\right\rangle,\left[p_{3}, p_{2}\right\rangle\right)$.

Proof. If $(M,\|\circ\|)$ is euclidean, any $\mathcal{C}$-orthocentric system $\left\{p_{1}, p_{2}, p_{3}, p_{4}\right\}$, with $p_{4}$ on the line containing $A_{B}\left(\left[p_{3}, p_{1}\right\rangle,\left[p_{3}, p_{2}\right\rangle\right)$, holds the equality $\left\|p_{3}-p_{1}\right\|=\left\|p_{3}-p_{2}\right\|$.

Conversely, let $x, y \in(M,\|\circ\|)$ with $x \perp_{I} y$. By the Lemma (2.1) we only have to prove that there exist $t>1$ such that $x \perp_{I} t y$. Clearly it exists such $t$ if $x=O$ or $y=O$. Now, let $x, y \in M \backslash\{O\}$ with $x \perp_{I} y$. Define $\lambda=\|x+y\|$ and

$$
p_{4}=y \quad p_{3}=-y \quad x_{1}=x \quad x_{2}=-x
$$

By (5) from Lemma (3.5) we have a $\mathcal{C}$-orthocentric system $\left\{p_{1}, p_{2}, p_{3}, p_{4}\right\}$ such that $p_{4} \in A_{B}\left(\left[p_{3}, p_{1}\right\rangle,\left[p_{3}, p_{2}\right\rangle\right)$. Since $\left\|p_{3}-p_{1}\right\|=\left\|p_{3}-p_{2}\right\|$, and the definition of Busemann angular bisectors, then

$$
A_{B}\left(\left[p_{3}, p_{1}\right\rangle,\left[p_{3}, p_{2}\right\rangle\right)=\left[p_{3}, \frac{p_{1}+p_{2}}{2}\right\rangle
$$

Thus, by (3) from Lemma 3.5 , we have $p_{1}=\frac{\lambda y}{\|y\|}-x$ and $p_{2}=\frac{\lambda y}{\|y\|}+x$. Then,

$$
\left\|x-\left(1+\frac{\lambda}{\|y\|}\right) y\right\|=\left\|p_{3}-p_{1}\right\|=\left\|p_{3}-p_{2}\right\|=\left\|x+\left(1+\frac{\lambda}{\|y\|}\right) y\right\|
$$

and therefore $x \perp_{I} t y$, taking $t=1+\frac{\lambda}{\|y\|}>1$. 


\section{References}

[1] AMIR, D. (1986) Characterizations of Inner Product Spaces. Birkhüser. Basel.

[2] ALONSO, J. (1994) “Uniqueness properties of isosceles orthogonality in normed linear spaces", Ann. Sci. Math. Quebec. Vol. 18 (1), 25 - 38.

[3] ASPLUND, E y GRÜNBAUM, B. (1960) "On the geometry of Minkowski planes". Enseign. Math. Vol. 6, 299-306.

[4] BUSEMANN, H. (1975) Planes with analogues to Euclidean angular bisectors. Math. Scand. Vol. 36, 5-11.

[5] DÜVELMEYER, N. (2004) "Angle Measures and Bisectors in Minkowski Planes". Canadian Mathematical Bulletin. Vol. 48, 523-534.

[6] JOHnSON, R. A. (2007) Advanced Euclidean Geometry, Dover Publications, Inc., Mineola, New York.

[7] MARTINI, H, SWANEPOEL, K. J y WEIß, G. (2001) "The Geometry of Minkowski Spaces - A Survey. Part I". Expositiones Math. Vol. 19, 97-142.

[8] MARTINI, H and SPIROVA, M. (2007) "The Feuerbach Circle and Orthocentricity in Normed Planes". L'Enseignement Mathematique. Vol. 53 (2), 237-258.

[9] MARTINI, H and SWANEPOEL, K. J. (2004) "The Geometry of Minkowski Spaces - A Survey. Part II". Expositiones Math. Vol. 22, 93-144.

[10] MARTINI, H y WU, S. (2009) "On Orthocentric Systems in Strictly Convex Normed Planes". Extracta Mathematicae. Vol. 24 (1), 31-45.

[11] THOMPSON, A. C. (1996) Minkowski Geometry. Encyclopedia of Mathematics and Its Applications. Vol. 63. Cambridge University Press. Cambridge.

[12] WU, S. (2009) "Geometry of Minkowski Planes and Spaces - Selected Topics".Chemnitz University of Technology. Tesis Doctoral. 